\title{
The decay of cyclonic eddies by Rossby wave radiation
}

\author{
By N. ROBB MCDONALD \\ Department of Mathematics, University College London, Gower Street, London WC1E 6BT UK
}

(Received 3 February 1997 and in revised form 4 December 1997)

It is argued that because shallow water cyclones on a $\beta$-plane drift westward at a speed equal to an available Rossby wave phase speed, they must radiate energy and cannot, therefore, be steady. The form of the Rossby wave wake accompanying a quasi-steady cyclone is calculated and the energy flux in the radiated waves determined. Further, an explicit expression for the radiation-induced northward drift of the cyclone is obtained. A general method for determining the effects of the radiation on the radius and amplitude of the vortex based on conservation of energy and potential vorticity is given. An example calculation for a cyclone with a 'top-hat' profile is presented, demonstrating that the primary effect of the radiation is to decrease the radius of the vortex. The dimensional timescale associated with the decay of oceanic vortices is of the order of several months to a year.

\section{Introduction}

Observations of the Earth's ocean and also of the atmosphere's of Jupiter and Saturn show that there is a predominance of anticyclones over cyclones (see e.g. McWilliams 1985; Nezlin \& Sutyrin 1994). This asymmetry is also evident in non-quasi-geostrophic $\beta$-plane numerical experiments such as those performed by Cushman-Roisin \& Tang (1990). In particular, they demonstrated that anticyclones emerge in preference to cyclones from an initially turbulent field. Furthermore, experiments performed by Nezlin and co-workers (see Nezlin \& Sutyrin 1994 for a review) in a rotating paraboloid also demonstrated the longevity of anticyclones relative to cyclones. The present work explores the possibility that the asymmetry may be due to the differing westward propagation speeds of cyclones and anticyclones. This is not a new idea. Indeed Nezlin \& Sutyrin (1994) suggested that the asymmetry in the dispersive and nonlinear properties of cyclones and anticyclones was one of the essential reasons for the observed predominance of anticyclones. Nycander (1994) develops the idea further and advocates a necessary condition for the existence of steady vortex structures, namely that the centre-of-mass speed of the vortex lies outside the range of possible linear wave phase speeds. Otherwise the vortex will resonate with the linear wave field, radiate energy (sometimes referred to as Cerenkov radiation), and subsequently decay. Nycander illustrates the idea with examples from geophysical fluid dynamics and magnetohydrodynamics where he compares the drift velocity of the vortex obtained by global integration methods with the phase velocities of the waves calculated from the linear dispersion relation. The purpose of the present work is to calculate analytically the Rossby wave field accompanying a cyclone on a $\beta$-plane, the associated energy loss, the meridional velocity and the response of the cyclone to this energy loss. 
Before proceeding, it should be noted that the presence of a background potential vorticity gradient (i.e. the $\beta$-effect) is not an essential ingredient for the production of cyclone/anticyclone asymmetry. For example, the shallow water $f$-plane numerical experiments of Polvani et al. (1994) clearly demonstrate the preference for the emergence of anticyclones over cyclones from an initially turbulent field. Polvani et al. (1994) speculate that the necessary asymmetry is provided by the different structural properties (e.g. different local deformation radius) of finite-amplitude shallow water cyclones and anticyclones. It should also be noted that the evolving turbulence experiments of Cushman-Roisin \& Tang (1990) involve strong vortex-vortex interactions and the wave-vortex mechanism to be explored here may not be the primary mechanism responsible for the cyclone-anticyclone asymmetry observed in their experiments.

The non-dimensional equations governing the motion of an eddy in a one-layer reduced-gravity fluid on the $\beta$-plane are

$$
\begin{gathered}
\boldsymbol{u}_{t}+(\boldsymbol{u} \cdot \nabla) \boldsymbol{u}+\boldsymbol{k}(1+\hat{\beta} y) \times \boldsymbol{u}=-\nabla \eta, \\
\eta_{t}+\nabla \cdot[(1+\eta) \boldsymbol{u}]=0,
\end{gathered}
$$

where $\boldsymbol{u}$ is the velocity field and $\eta$ is the displacement of the free surface from the background depth of unity. Variables have been made non-dimensional using the inverse of the Coriolis parameter $f$ as the timescale, and the deformation radius $L=\left(g^{\prime} H\right)^{1 / 2} / f$ as the lengthscale, where $g^{\prime}$ is the reduced gravity and $H$ is the undisturbed layer depth. The non-dimensional parameter $\hat{\beta}$ is given by $\hat{\beta}=\beta L / f$. The lengthscale, assumed to be the Rossby radius, is representative of many geophysical vortices and is used throughout the present work.

An example demonstrating the differing westward propagation speed $U$ of cyclones and anticyclones, derived by Cushman-Roisin, Chassignet \& Tang (1990) assuming small Rossby number and timescale (see their equation 15), is given by the formula:

$$
U=-\hat{\beta}\left[1+\frac{1}{2} \frac{\iint \eta^{2} \mathrm{~d} A}{\iint \eta \mathrm{d} A}\right] .
$$

In dimensional terms the zonal velocity scales with the long Rossby wave speed, i.e. $\beta L^{2}$. The formula is accurate to a relative error of order the Rossby number. Moreover the meridional (north-south) velocity vanishes to the same degree of accuracy. Following Cushman-Roisin et al. (1990) an eddy for which $\eta>0$ (a localized thickening of the fluid) is called an anticyclone and an eddy for which $\eta<0$ (a localized depression) is called a cyclone. It will also be assumed that the eddy is a monopole and that $\eta$ does not change sign over the extent of the eddy.

The key observation regarding (2) is that if $\eta>0$ the centre of mass of the eddy travels at velocity $c<-\hat{\beta}$ and if $\eta<0$ the centre of mass of the eddy travels at velocity $c>-\hat{\beta}$. In fact, for a cyclone, since the minimum value of $\eta$ is -1 , Cushmann-Roisin et al. (1990) show that (2) implies $-\hat{\beta}<c<-\hat{\beta} / 2$. This difference in the westward drift velocities of cyclones and anticyclones is not present in the quasi-geostrophic equations which assume that the free surface displacement is infinitesimally small. In particular, in quasi-geostrophic theory, both cyclones and anticyclones propagate westward at the same speed, namely the long Rossby wave speed. Thus, crucial to the present work is the requirement that vortex amplitudes be finite, i.e. not quasi-geostrophic. 
Recall that free linear Rossby waves for a one-layer reduced-gravity fluid governed by (1) have phase velocities $c_{p}$ such that

$$
-\hat{\beta}<c_{p}<0 .
$$

Importantly, anticyclones according to (2) have a westward drift speed outside the range of linear Rossby wave phase speeds. In contrast, cyclones drift with a speed which matches a possible Rossby wave phase speed. Thus, in the case of cyclones, an exterior wave field must accompany the propagating eddy and they can no longer be considered to be steady.

Recently, Benilov (1996) has obtained a formula similar to (2) for the westward drift speed of a nonlinear shallow water eddy using a perturbation method based on the smallness of $\hat{\beta}$. His formula, valid for order-one Rossby numbers, also demonstrates the property that cyclones have westward drift velocity which matches available Rossby wave speeds, while anticyclones, on the other hand, drift westward faster than all Rossby wave phase speeds. Both (2) and Benilov's result are consistent with the interpretation that coherent structures on a $\beta$-plane drift westward at the 'local' long Rossby wave speed. In particular, anticyclones have a greater local deformation radius (owing to larger depth), and hence have greater long Rossby wave speed than the surrounding fluid. Conversely, cyclones have a deformation radius smaller than the ambient deformation radius and therefore have a smaller long Rossby wave speed than the surrounding fluid. It is this property of differing westward drift speeds of cyclones and anticyclones that is taken as the starting point for the present work.

A notable study of radiating vortex structures on a $\beta$-plane involving a single active layer of fluid is that by Flierl \& Haines (1994). Starting with quasi-geostrophic theory they studied the radiative response of a modon which was forced to propagate at a velocity which was resonant with a Rossby wave. Similar to the present work they calculated the energy flux in the quasi-steady Rossby wave field and found that the leading-order response of the modon was to decrease its radius. The present work also has similarities to Korotaev \& Fedotov (1994) who studied an intense barotropic (rigid-lid) monopole on the $\beta$-plane. Again the westward drift implies the generation of a resonant Rossby wave field. Korotaev \& Fedotov showed that the radiation implied a meridional drift in the vortex which also lead to a decrease in the vortex radius. Numerical evidence was presented which qualitatively confirmed their theoretical results.

The aim of this work is to calculate the form of the exterior Rossby wave field for a shallow water cyclone in the quasi-steady limit and also the associated energy flux in this wave field. By quasi-steady it is meant that the time-dependent parameters of the system (specifically, the eddy radius and depth) are assumed to vary slowly on a Rossby wave timescale, i.e. the time taken for a Rossby wave to propagate the characteristic length of the vortex. This implies, for the purposes of calculating the exterior Rossby wave field, that the vortex may be considered steady. This is justified a posteriori. The flux of energy implies, of course, that the cyclone cannot be steady but must lose energy and decay. The timescale and form of this decay is to be calculated for a specific ('top-hat') eddy profile.

The plan of the paper is as follows: In $\S 2$ the main assumptions are stated, the governing equation for the exterior of the vortex derived and the boundary conditions to which it is subject to are discussed. Section 3 analyses the non-radiating case of an anticyclone. Section 4 derives the Rossby wave field associated with a cyclone and the associated meridional drift. The radiative loss of energy of the cyclone is calculated 
in $\S 5$. Coupled with the Lagrangian conservation of potential vorticity a method enabling the response of the cyclone to Rossby wave radiation is also presented in $\S 5$. In $\S 6$ an example calculation for the decay of an idealized cyclone with 'top-hat' depth profile suggests that the wave decay mechanism may be of significance on geophysical timescales. The work is concluded in $\S 7$.

\section{Problem formulation}

The following assumptions are made.

(i) The eddy propagates in a westward direction with speed $U=\hat{\beta} u$ where $\hat{\beta} \ll 1$ and $u<-1$ for anticyclones and $-1<u<0$ for cyclones. For oceanic eddies, typically $\hat{\beta} \approx 0.002-0.01$. This is the only small parameter required for the theory developed in $\S \S 2-5$. The possibility of motion in the meridional $(y)$ direction is allowed for; it is not prescribed but, rather, determined as consequence of the radiative process. However, it is assumed that the meridional velocity $V$ is small relative to the zonal velocity, i.e. $|V / U| \ll 1$.

(ii) The eddy is intense. That is, the ratio of the eddy turnover time to the wave propagation time is small. The intense assumption implies that the vortex itself does not disperse into Rossby waves, but rather nonlinear effects are sufficiently large to cause the vortex to propagate as a whole transporting fluid within a closed separatrix of the field $\eta+U y$. Reznik (1992) discusses similar separatrix formation for intense, but admittedly singular, quasi-geostrophic vortices.

(iii) To leading order the eddy is assumed radially symmetric and remains so throughout its evolution. That is $\eta=\eta(r)$. To facilitate analytical progress, the eddy boundary (i.e. the separatrix) is assumed to be a circle of radius $a$, which may be a slowly varying function of time.

At the separatrix $(r=a)$ the orbital (circulative) velocity of the vortex must be of the same order as the drift velocity, namely $O(\hat{\beta})$. Since this orbital velocity is small, this implies that the flow here (and beyond) is, to leading order, geostrophic. Hence, by $(1 a), \eta=O(\hat{\beta})$ at $r=a$. Writing $\eta=\hat{\beta} \psi$, where $\psi$ is the geostrophic streamfunction, it follows that the condition on the separatrix is

$$
\psi+u y=C \quad \text { on } \quad r=a,
$$

where $C$ is some constant to be determined. Note that the separatrix is essentially the matching region between the interior flow where the swirl velocity of the vortex is much greater than the drift velocity and the far-field velocity where the swirl velocity is very much weaker than the drift velocity.

In a frame of reference moving zonally with the vortex at velocity $\hat{\beta} u$ the time derivatives in $(1 a, b)$ can be replaced by $-\hat{\beta} u \partial / \partial x$ where smaller time derivatives have been neglected through the quasi-steady assumption. Standard expansion of the shallow water equations $(1 a, b)$ in the parameter $\hat{\beta}$ (i.e. putting $\boldsymbol{u}=\hat{\beta} \boldsymbol{u}$ and $\eta=\hat{\beta} \psi$ in $(1 a, b))$ reveals that the small parameter is precisely the correct location for derivation of the quasi-geostrophic equation for $\psi$. Note that, as in Benilov (1996), $\hat{\beta}$ is playing the role of the Rossby number since the swirl velocity of the vortex is of this order at the separatrix. For $r \geqslant a, \psi$ thus satisfies

$$
u \psi_{x}-u \nabla^{2} \psi_{x}+J\left[\psi, \nabla^{2} \psi\right]+\psi_{x}=0,
$$

where $J[f, g]=f_{x} g_{y}-f_{y} g_{x}$ is the Jacobian. The essential point is that outside the separatrix the flow is quasi-geostrophic. Benilov (1996) also derives this quasi- 
geostrophic relation at the periphery of a shallow water vortex and beyond. The nonlinear dynamics inside the separatrix are unspecified and are only important to the following insofar as they determine the westward drift velocity $u$ of the vortex, i.e. through the boundary condition (4).

As in Korotaev \& Fedotov (1994) it is assumed that the separatrix has only one saddle point which is located in the upper half-plane for a cyclone and the lower half-plane for an anticyclone. This assumption is born from the realization that flow around a vortex is more like flow around a cylinder with circulation than without. The forcing of a single stagnation point to the north or south of the vortex is an attempt to model this feature and is clearly more realistic for monopoles than the symmetrical fore and aft stagnation points that occur, for example, in the case of a modon. The condition that forces this topology can be written as

$$
\psi_{y}=-u, \quad r=a,\left\{\begin{array}{lll}
\theta=\pi / 2, & & \text { cyclone } \\
\theta=-\pi / 2, & & \text { anticyclone. }
\end{array}\right.
$$

Finally, it is assumed that

$$
\psi \rightarrow 0 \quad \text { as } \quad r \rightarrow \infty .
$$

The mathematical task is to solve (5) subject to conditions (4), (6) and (7). For the case of a cyclone, as will be seen, these conditions need to be supplemented by a radiation condition.

Note that (5) can be written as

$$
J\left[\psi+u y, \nabla^{2} \psi+(u+1) y\right]=0,
$$

which has solution

$$
\nabla^{2} \psi+(u+1) y=F(\psi+u y),
$$

where $F($.$) is some arbitrary differentiable function. A suitable choice for F$ for which (9) becomes homogeneous, and consistent with the linear version of (5) (which is the expected form of (5) in the far field), is $F(z)=(u+1) z / u$.

For this choice of $F(z), \psi$ satisfies

$$
\nabla^{2} \psi-\frac{(u+1)}{u} \psi=0 .
$$

Benilov (1996) also obtains this equation for the periphery of the vortex and beyond. It follows from (2) that for an anticyclone $(u+1) / u>0$ and for a cyclone $(u+1) / u<0$.

\section{Anticyclone}

For completeness, and to contrast the forthcoming case of a cyclone, an analysis of an anticyclone is done first. By the arguments presented in $\S 1$ this should be a robust object since its westward drift velocity lies outside the range of possible Rossby wave phase speeds.

Let $b^{2}=(u+1) / u$, so that $(10)$ becomes

$$
\nabla^{2} \psi-b^{2} \psi=0 .
$$

The solution of (11) satisfying (4) and (7) is

$$
\psi=C \frac{K_{0}(b r)}{K_{0}(b a)}-u a \frac{K_{1}(b r)}{K_{1}(b a)} \sin \theta .
$$




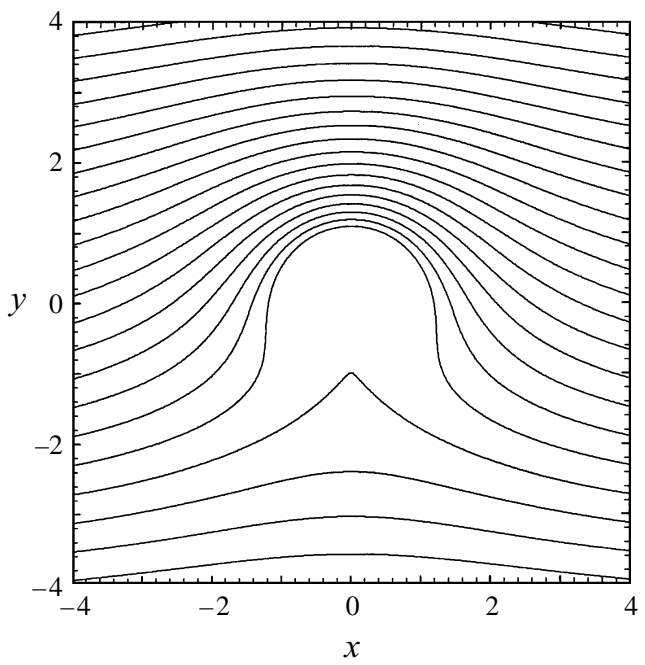

Figure 1. Contours of $\psi+u y$ for an anticyclone in the region $r \geqslant a$ where $a=1$ and $u=-1.1$. The contour interval is 0.4 .

The constant $C$ is determined from the stagnation point condition (6):

$$
C=-u a \frac{K_{0}(b a) K_{2}(b a)}{\left[K_{1}(b a)\right]^{2}},
$$

which is always positive implying that the sense of the circulation, $\psi_{r}$, at $r=a$ is negative, as expected for an anticyclone. Substituting (13) in (12) gives $\psi$ :

$$
\psi=-\frac{u a}{K_{1}(b a)}\left[\frac{K_{2}(b a)}{K_{1}(b a)} K_{0}(b r)+K_{1}(b r) \sin \theta\right] .
$$

A plot of $\psi$ given by (14) for $r \geqslant a$ is shown in figure 1. It is evident that the streamfunction is evanescent for $r \geqslant a$ and the streamfunction $\psi$ decays exponentially as $r \rightarrow \infty$. The energy of an anticyclone remains localized and it is expected, at least with respect to the (non)radiation of energy, that anticyclones are robust, long-lived features.

\section{Cyclone}

Let $b^{2}=-(u+1) / u$, so that (10) becomes

$$
\nabla^{2} \psi+b^{2} \psi=0 .
$$

For this case conditions (4) and (7) need to be supplemented by an appropriate radiation condition which precludes upstream waves, namely

$$
\lim _{r \rightarrow \infty} r^{1 / 2} \psi(r, \theta)=0, \quad \pi / 2 \leqslant \theta \leqslant 3 \pi / 2 .
$$

The solution is constructed in a similar manner to Miles (1968) (see also Swaters \& Flierl 1991 and Swaters 1994) with the additional allowance for terms which are symmetric about the $x$-axis such as occur in Flierl (1984) and Korotaev \& Fedotov (1994) (i.e. terms involving $\cos n \theta$ ). These terms are necessary in order to satisfy the 
saddle point condition (6) on the separatrix. To proceed, write $\psi$ as

$$
\psi=\sum_{n=1}^{\infty} \alpha_{n}\left[Y_{n} \sin n \theta+h_{n}(r, \theta)\right] / Y_{n}(b a)+\sum_{n=0}^{\infty} \gamma_{n}\left[Y_{n} \cos n \theta+g_{n}(r, \theta)\right] / Y_{n}(b a),
$$

where

$$
\begin{aligned}
& h_{n}(r, \theta)=\sum_{m=1}^{\infty} \beta_{n, m} J_{m}(b r) \sin m \theta, \\
& g_{n}(r, \theta)=\sum_{m=0}^{\infty} \epsilon_{n, m} J_{m}(b r) \cos m \theta .
\end{aligned}
$$

Using the asymptotic forms for $J_{n}$ and $Y_{n}$ as $r \rightarrow \infty$ it follows from (16), (17) and $(18 a, b)$ that

$$
\begin{gathered}
\left.\sin (2 n \theta)=\sum_{m=0}^{\infty}(-1)^{m+n+1} \beta_{2 n, 2 m+1} \sin [(2 m+1) \theta)\right] \\
\sin [(2 n+1) \theta]=\sum_{m=0}^{\infty}(-1)^{m+n} \beta_{2 n+1,2 m} \sin (2 m \theta),
\end{gathered}
$$

and

$$
\begin{gathered}
\left.\cos (2 n \theta)=\sum_{m=0}^{\infty}(-1)^{m+n+1} \epsilon_{2 n, 2 m+1} \cos [(2 m+1) \theta)\right], \\
\cos [(2 n+1) \theta]=\sum_{m=0}^{\infty}(-1)^{m+n} \epsilon_{2 n+1,2 m} \cos (2 m \theta) .
\end{gathered}
$$

The slow $r^{-1 / 2}$ decay of $J_{n}$ and $Y_{n}$ as $r \rightarrow \infty$ implies that the exterior field has infinite energy if, and only if, it extends to infinite radius. However the scenario to bear in mind here is an initial value problem where, for large times after some initial adjustment period, the wave field of the form (17) extends to large, but finite, $r$ at a position given by $r=c_{g} t$ where $c_{g}$ is the radial group velocity. Ahead of this wavefront the streamfunction amplitude vanishes. Thus the energy is finite in the exterior and the relevant quantity being sought is the energy flux in the wave field.

Equations $(19 a, b)$ are the same as obtained by Swaters (1994) and have solution

$$
\beta_{n, m}= \begin{cases}\frac{4}{\pi} \frac{n}{m^{2}-n^{2}}, & m \text { odd, } n \text { even } \\ \frac{4}{\pi} \frac{m}{m^{2}-n^{2}}, & n \text { odd, } m \text { even } \\ 0, & m+n \text { even },\end{cases}
$$

where 0 is considered even for the purposes of the above calculation. Similarly the solution to $(20 a, b)$ can be obtained:

$$
\epsilon_{n, m}= \begin{cases}\frac{4}{\pi} \frac{m}{m^{2}-n^{2}}, & \text { modd, } n \text { even } \\ \frac{4}{\pi} \frac{n}{m^{2}-n^{2}}, & n \text { odd, } m \text { even } \\ 0, & m+n \text { even. }\end{cases}
$$




$\begin{array}{lcc} & a b=1.0 & a b=0.2 \\ \gamma_{0} / C & -0.146 & 0.957 \\ \gamma_{1} / C & 0.917 & 0.112 \\ \gamma_{2} / C & 0.057 & 7.14 \times 10^{-5} \\ \gamma_{3} / C & 0.0142 & 6.24 \times 10^{-5} \\ \gamma_{4} / C & 2.5 \times 10^{-4} & 1.19 \times 10^{-8}\end{array}$

TABLE 1 . The first five values of $\gamma / C$ with zero meridional velocity for two cases: $a b=1.0$ and $a b=0.2$.

It remains to satisfy the boundary condition (4) on $r=a$, namely

$$
\left.\psi\right|_{r=a}=-u a \sin \theta+C,
$$

where $C$ is some constant to be determined using the stagnation point condition at $r=a$ and $\theta=\pi / 2$. Substitution of (17) into (23) gives (cf. Swaters 1994)

$$
\sum_{n=1}^{\infty} \alpha_{n} \Gamma_{n, m}(a b)=-u a \delta_{m 1}, \quad \sum_{n=0}^{\infty} \gamma_{n} \Gamma_{n, m}^{\prime}(a b)=C \delta_{m 0}
$$

where

$$
\Gamma_{n, m}=\delta_{n m}+\beta_{n, m} \frac{J_{m}(b a)}{Y_{n}(b a)}, \quad \Gamma_{n, m}^{\prime}=\delta_{n m}+\epsilon_{n, m} \frac{J_{m}(b a)}{Y_{n}(b a)} .
$$

Note that equations $(23 a, b)$ and $(24 a, b)$ represent two sets of infinite-dimensional matrix equations for the unknown vectors $\alpha=\left(\alpha_{1}, \alpha_{2}, \cdots\right)$ and $\gamma=\left(\gamma_{0}, \gamma_{1}, \cdots\right)$. Note that the set (23a) and (24a) is precisely that solved by Swaters (1994). He points out that as $n \rightarrow \infty, \Gamma_{n, m}(a b) \approx \delta_{n, m}$ which implies that relatively few terms of the matrix $\Gamma_{n, m}$ are needed for very accurate results. Indeed, he used the leading $20 \times 20$ finite systems of equations to show that for $a=b=1, \alpha_{1} \approx-0.97 u a, \alpha_{2} \approx-0.12 u a$, $\alpha_{3} \approx-7.3 \times 10^{-4} u a$, etc. For $a \ll 1$ convergence is even more rapid owing to property that $\Gamma_{n, m}(a b) \approx \delta_{n, m}$ as $a \rightarrow 0$ for all $n, m$. For example, if $a=0.2$ and $b=1$ solving the leading $20 \times 20$ system of equations gives $\alpha_{1} \approx-0.999997 u a, \alpha_{2} \approx-1.2 \times 10^{-3} u a$, $\alpha_{3} \approx-3.3 \times 10^{-9} u a$, etc. As will be seen, the small- $a$ limit is of practical significance, since the main response of the cyclone to the radiation of Rossby waves is a decrease in radius. Thus, for practical purposes such as calculating the energy radiated by the Rossby waves, we shall take $\alpha_{1}=-u a$ and $\alpha_{m}=0$ for $m \geqslant 2$.

Similarly, $(24 b)$ and $(25 b)$ also have the property that $\Gamma_{n, m}^{\prime}(a b) \approx \delta_{n, m}$ as $n \rightarrow \infty$. Again sufficient accuracy is obtained by approximating the infinite set of equations by the leading $20 \times 20$ system. The behaviour is illustrated in table 1 where the first five values of $\gamma_{i}$ are given for $a=b=1.0$ and $a=0.2, b=1.0$. Note however that the presence of the $\cos \theta$ term at $r=a$ in (17) is suggestive of possible meridional motion. More precisely, a $\cos \theta$-like dipole, which has an axis of antisymmetry aligned in the meridional direction, will cause the eddy to move in that direction. The calculations presented in table 1 were obtained by requiring the meridional motion to vanish, i.e. the right-hand side of $(24 b)$ for the equation involving $\cos \theta$ vanishes since there is no $\delta_{m 1}$ term. There is no reason, however, to a priori preclude the possibility of meridional motion. Indeed, numerical experiments on eddy evolution frequently show meridional motion (see, for example, Davey \& Killworth 1984). Here we postulate 


$\begin{array}{lcc} & a b=1.0 & a b=0.2 \\ \gamma_{0} / C & 0.9992 & 1.0 \\ \gamma_{1} / C & 4.8 \times 10^{-3} & 1.26 \times 10^{-7} \\ \gamma_{2} / C & 1.7 \times 10^{-3} & -3.08 \times 10^{-10} \\ \gamma_{3} / C & -9.3 \times 10^{-2} & 6.52 \times 10^{-5} \\ \gamma_{4} / C & 2.05 \times 10^{-5} & 2.45 \times 10^{-13}\end{array}$

TABLE 2. The first five values of $\gamma / C$ with non-zero meridional velocity given by (27) for two cases: $a b=1.0$ and $a b=0.2$.

the existence of meridional motion and, instead of $(24 b)$, solve the modified equation

$$
\sum_{n=0}^{\infty} \gamma_{n} \Gamma_{n, m}^{\prime}(a b)=C \delta_{m 0}+v a \delta_{m 1}
$$

where $v$ is the meridional velocity which, for the moment, is undetermined. To compensate for the extra degree of freedom due to meridional motion it is assumed that most of the energy in the radiated wave field exists in the lowest-order mode disturbance $\gamma_{0}$ (that is $\gamma_{i} \ll \gamma_{0}$ for $i \geqslant 1$ ). Some justification for this comes from the solution of $(24 a)$ where it was demonstrated that most of the energy occurs in the lowest-order (i.e. $\sin \theta$ ) mode. Further justification is that it yields an explicit formula for the meridional velocity which reduces to a known result in the barotropic limit (see below). With this assumption it follows from (26) with $m=1$ that

$$
v a=\gamma_{0} \Gamma_{0,1}^{\prime}=\gamma_{0} \frac{4}{\pi} \frac{J_{1}(b a)}{Y_{0}(b a)} .
$$

Equations (26) and (25b) are then solved with $v$ given by (27). The results are displayed in table 2 . It is evident that now convergence is now extremely rapid and that indeed, as assumed, most of energy in the radiated field exists in the lowest mode $\gamma_{0}$ and, further, $\gamma_{0}=C$. Note that the original assumption $\gamma_{i} \ll \gamma_{0}$ is only explicitly used in the (26) with $m=1$ and the subsequent infinite-dimensional system gives a solution consistent with this assumption.

It remains to determine $C$. As in the case of the anticyclone this is done by invoking the stagnation point condition (6), namely that $\psi_{y}=-u$ on $(r, \theta)=(a, \pi / 2)$. Note that at this point $\cos [(2 m+1) \theta]=\sin [2 m \theta]=0$ as well as $\theta_{y}=0$ and $r_{y}=1$. Thus differentiating (17) with respect to $y$ and using (6) and the approximations $\alpha=(-u a, 0,0, \cdots)$ and $\gamma=(C, 0,0, \cdots)$ yields

$$
C=\gamma_{0}=u a \frac{Y_{0}(b a) Y_{2}(b a)}{\left[Y_{1}(b a)\right]^{2}}
$$

Substituting (28) into (27) finally gives a determinate expression for $v$ :

$$
v=\frac{4}{\pi} u \frac{J_{1}(b a) Y_{2}(b a)}{\left[Y_{1}(b a)\right]^{2}} .
$$

Before illustrating the general behaviour of $v$ given by (29) consider first the limit $a \rightarrow 0$. Physically this corresponds to a vortex whose lengthscale is much less than the deformation radius. This is equivalent to the barotropic limit. Asymptotic 


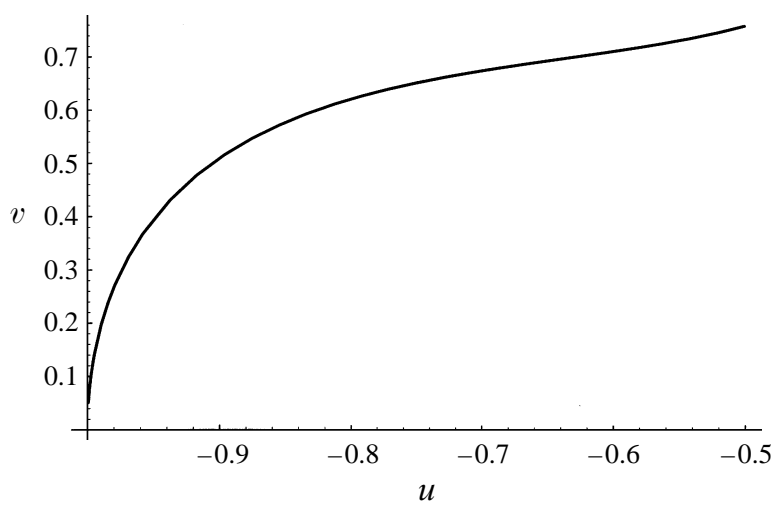

FIGURE 2. Plot of $v$ as a function of $u$ where $v$ is given by (29) and $b^{2}=-(u+1) / u$ for $-1<u<-1 / 2$ and $a=1$.

expressions of the Bessel functions in (29) for small arguments give the leading-order expression

$$
v \approx-2 u a b .
$$

The limiting result (30) agrees exactly with the result obtained by Korotaev \& Fedotov (1994) $\dagger$ who considered the motion of a barotropic vortex on a $\beta$-plane.

Choosing $a=1$, a plot of $v$ as a function of $u$ is shown in figure 2. Note that $v>0$, i.e. the cyclone moves north as a result of the radiation. The magnitude of the meridional velocity decreases as the westward drift velocity approaches -1 , i.e. the quasi-geostrophic limit. That is, smaller-amplitude cyclones have smaller northward velocities. Observe that for much of the range of $u, v$ is of the same order of magnitude as $u$. This violates the assumption of the preceding analysis that $|v / u| \ll 1$ (see assumption (i) in $\S 2$ ). If the magnitudes of $v$ and $u$ are of comparable order the governing equation (4) would contain terms involving $v$ and derivatives with respect to $y$. Moreover, it seems likely that the saddle point would no longer be at $\theta=\pi / 2$, if there were to be significant meridional motion. Strictly speaking the result (29) is valid only when $u$ is less than, say, -0.9 where the approximation $|v / u| \ll 1$ is valid. Note for such values of $u, b \ll 1$ and so for cyclones of $O(1)$ radius it follows that $a b \ll 1$. This is precisely the limit for which the assumption that most of the radiated energy resides in the gravest mode holds true. It is evident therefore that the assumption $|v / u| \ll 1$ is consistent with the assumption that most of the radiated energy resides in the gravest mode. Even with this caution, it is possible that (29) represents a wider range of $u$ values since the situation is reminiscent of Korotaev \& Fedotov (1994) who also made the assumption that $|v / u| \ll 1$, but found that their analysis predicted, for some parameter values, that $|v| \sim u$. Indeed they found that $v>u$ in some cases. Even for such parameter values they found good agreement between their theoretical predictions and numerical experiment.

If for the purpose of illustrating $\psi$ and calculating the energy flux in the wave-field we take, to a good approximation, $\boldsymbol{\alpha}=(-u a, 0,0, \cdots)$ and $\gamma=(C, 0,0, \cdots)$ where $C$ is

$\dagger$ In particular their equation (4.21). Note that there is a misprint in their equation: it should read $V=(\Gamma / 2 \pi) \lambda=2 R_{0}(-U)^{1 / 2}$. Here $a$ is equivalent to $R_{0}$ and $b$ is equivalent to $(-1 / U)^{1 / 2}$ in the barotropic limit. 
(a)

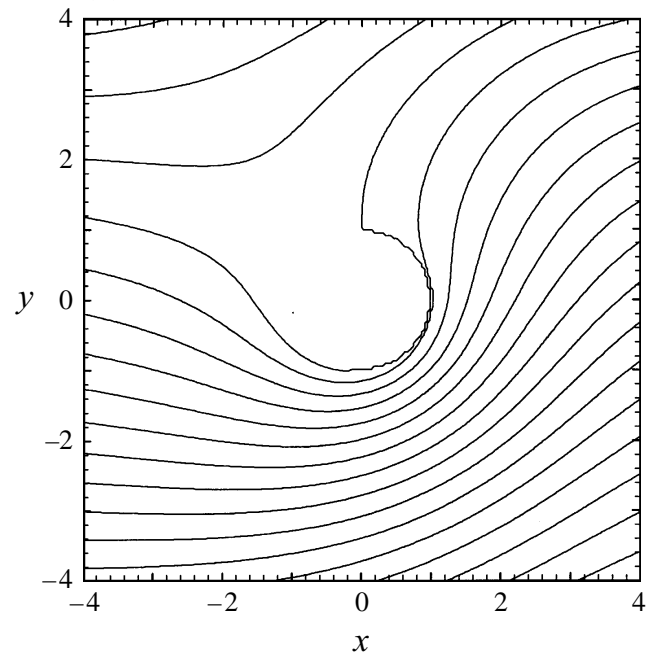

(b)

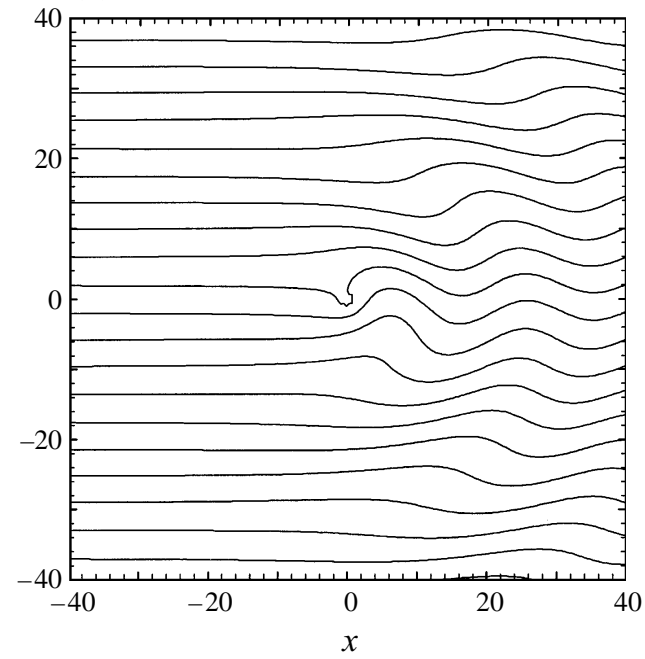

Figure 3. Contours of $\psi+u y$ for a cyclone for $(a)-4 \leqslant x, y \leqslant 4$ (contour interval of 0.5 ) and (b) $-40 \leqslant x, y \leqslant 40$ (contour interval of 3.5). In each case $b=1 / 3(u=-0.9)$ and $a=1$.

given by (28), (17) yields

$$
\begin{aligned}
\psi=u a \frac{Y_{2}(b a)}{\left[Y_{1}(b a)\right]^{2}}\left[Y_{0}(b r)+\frac{4}{\pi} \sum_{m=0}^{\infty} \frac{J_{2 m+1}(b r)}{2 m+1} \cos [(2 m+1) \theta]\right] \\
-\frac{u a}{Y_{1}(b a)}\left[Y_{1}(b r) \sin \theta+\frac{2}{\pi} \sum_{m=1}^{\infty} \frac{4 m^{2}}{4 m^{2}-1} J_{2 m}(b r) \sin [2 m \theta]\right] .
\end{aligned}
$$

This expression contains terms similar to Flierl \& Haines (1994) and also Swaters (1994). Sketches of the streamfunction given by (31) are given in figure $3(a, b)$. The downstream waves are clearly evident as is the location of the saddle, or stagnation, point on the northern side of the vortex.

\section{The cyclone decay}

As $r \rightarrow \infty$, using standard asymptotic results for Bessel functions, (31) becomes

$$
\psi= \begin{cases}2 A\left(\frac{2}{\pi b r}\right)^{1 / 2} \sin \left(b r-\frac{\pi}{4}\right)+2 C\left(\frac{2}{\pi b r}\right)^{1 / 2} \cos \left(b r-\frac{\pi}{4}\right) \sin \theta, & x>0 \\ 0, & x<0,\end{cases}
$$

where

$$
A=u a \frac{Y_{2}(b a)}{\left[Y_{1}(b a)\right]^{2}}, \quad C=\frac{u a}{Y_{1}(b a)} .
$$

The leading-order energy density $\rho=\left(\psi_{r}^{2}+\left(1 / r^{2}\right) \psi_{\theta}^{2}+\psi^{2}\right) / 2$ of the waves in (32) as $r \rightarrow \infty$, after averaging the cyclic terms over one period, is

$$
\rho=\frac{2\left(1+b^{2}\right) u^{2} a^{2}\left[Y_{2}(b a)\right]^{2}}{\pi b r\left[Y_{1}(b a)\right]^{4}}\left[1+\frac{\left[Y_{1}(b a)\right]^{2}}{\left[Y_{2}(b a)\right]^{2}} \sin ^{2} \theta\right] .
$$


In a frame of reference moving with zonal velocity $u$, linear quasi-geostrophic Rossby waves have dispersion relation

$$
\omega(\kappa, \theta)=-u \kappa \cos \theta-\frac{\kappa \cos \theta}{1+\kappa^{2}},
$$

where $\kappa$ is the radial wavenumber and $k=\kappa \cos \theta$ and $l=\kappa \sin \theta$ are the zonal and meridional wavenumbers respectively. In this frame of reference the waves are stationary, i.e. $c_{p}=\omega / k=0$. Thus

$$
u=-\frac{1}{1+\kappa^{2}} .
$$

Recall that for a cyclone $b^{2}=-(u+1) / u$ and so, from (36), $\kappa^{2}=b^{2}$. The radial group velocity $c_{g}$ is

$$
c_{g}=\frac{\partial \omega}{\partial \kappa}=\frac{2 b^{2} \cos \theta}{\left(1+b^{2}\right)^{2}} .
$$

Note if $b=0$ then $c_{g}=0$ and no energy is radiated by linear waves. This corresponds to the case $u=-1$ and therefore, from (2), $\eta \equiv 0$, i.e. there is no eddy and so this case is ignored.

The rate at which energy is lost from the eddy can now be calculated (recalling that the non-dimensional amplitude of $\psi$ is $\hat{\beta}$ ):

$$
\begin{aligned}
\frac{\mathrm{d} E}{\mathrm{~d} t} & =-\hat{\beta}^{2} \int_{-\pi / 2}^{\pi / 2} \rho c_{g} r \mathrm{~d} \theta \\
& =-\hat{\beta}^{2} \frac{8 a^{2} b\left[Y_{2}(b a)\right]^{2}}{\pi\left(1+b^{2}\right)^{3}\left[Y_{1}(b a)\right]^{4}}\left[1+\frac{1}{3} \frac{\left[Y_{1}(b a)\right]^{2}}{\left[Y_{2}(b a)\right]^{2}}\right] .
\end{aligned}
$$

The smallness of the term on the right-hand side of (38) (i.e. order $\hat{\beta}^{2}$ ) justifies the quasi-steady approximation.

In general, the energy of the eddy depends on both its radius $a$ and its amplitude $\eta$ (which through (2) affects the magnitude of $u$ and, in turn, $b$ ) both of which are considered slowly varying quantities. Thus another equation in addition to (38) is required in order to close the system. It will be supposed that, in the spirit of Flierl \& Haines (1994), the potential vorticity of the fluid column at the centre of the eddy is conserved as it moves northward. Numerical experiments for monopole evolution on a quasi-geostrophic $\beta$-plane by Sutyrin et al. (1994) show that that fluid particles forming a central core of the eddy remain coherent throughout the evolution. Thus it seems reasonable to choose the central fluid column for application of potential vorticity conservation. Hence

$$
\frac{\mathrm{D}}{\mathrm{D} t}\left(\frac{\nabla^{2} h+1+\hat{\beta} y}{h}\right)=0
$$

where the term in the round brackets is the potential vorticity of the central fluid column. Equations (38) and (39) are sufficient to determine the evolution of cyclones in response to Rossby wave radiation. An example calculation is presented in the next section for the simple case of a cyclone with 'top-hat' profile. 


\section{An example of decay: a cyclone with 'top-hat' profile}

Consider a cyclone with depth profile

$$
\eta= \begin{cases}-\lambda, & r \leqslant a \\ 0, & r>a\end{cases}
$$

where $\lambda>0$. Although perhaps on over-simplified choice of profile it does represent the case when the leading-order energy is all potential and the leading-order potential vorticity is function of fluid depth only. Incidentally, such properties are shared by large-scale frontal geostrophic eddies where the relative vorticity can be ignored (see e.g. Cushman-Roisin 1986). For the purposes of calculating the effects of the radiative energy loss on the cyclone it is necessary to be able to calculate analytically the westward drift velocity $u$ given the profile (40). To do this, the formula (2) of Cushman-Roisin et al. (1990) is chosen.

The following properties are calculated from (2) and (40): $u=-1+\lambda / 2, b^{2}=$ $\lambda /(2-\lambda)$ and the energy (all potential) $E=\pi a^{2} \lambda^{2} / 2$. Using these quantities, and noting that $\nabla^{2} h=0$ for the central fluid column and $h=1-\lambda$ and $\mathrm{d} y / \mathrm{d} t=v$ the conservation of peak potential vorticity (39) leads to

$$
\begin{aligned}
\frac{\mathrm{d} \lambda}{\mathrm{d} t} & =-\hat{\beta} v(1-\lambda) \\
& =-\hat{\beta}^{2}(1-\lambda)(-1+\lambda / 2) \frac{4}{\pi} \frac{Y_{2}(b a) J_{1}(b a)}{\left[Y_{1}(b a)\right]^{2}}
\end{aligned}
$$

where the expression (29) has been used for $v$ and also the fact that $v=O(\hat{\beta})$. The energy equation (38) yields

$$
\lambda \frac{\mathrm{d} a}{\mathrm{~d} t}+a \frac{\mathrm{d} \lambda}{\mathrm{d} t}=-\hat{\beta}^{2} \frac{8 a b}{\pi^{2}\left(1+b^{2}\right)^{3} \lambda} \frac{\left[Y_{2}(b a)\right]^{2}}{\left[Y_{1}(b a)\right]^{4}}\left[1+\frac{1}{3} \frac{\left[Y_{1}(b a)\right]^{2}}{\left[Y_{2}(b a)\right]^{2}}\right],
$$

where $b^{2}=\lambda /(2-\lambda)$. The task is to solve the coupled system (41) and (42) for $a(t)$ and $\lambda(t)$ subject to given initial values of $a$ and $\lambda$. This is done numerically. In the numerical simulations it was noted that $\lambda$ remained essentially constant throughout, i.e. it stayed very close to its initial value. Comparison of the magnitude of the terms on the right-hand side of each of equations (41) and (42) shows that indeed $\mathrm{d} \lambda / \mathrm{d} t$ is relatively small compared to $\mathrm{d} a / \mathrm{d} t$. The implication is that the primary response of the cyclone to Rossby wave radiation is to lose energy by a decrease in radius rather than by a decrease in amplitude. This is consistent with the quasigeostrophic studies of Korotaev \& Fedotov (1994) and Sutyrin et al. (1994) who showed numerically that the vortex core (i.e. a region of 'trapped particles') shrinks during its evolution.

Results showing $a=a(\tau)$ and $\lambda=\lambda(\tau)$ are presented in figure $4 a, b$ for values of $\lambda$ equal to 0.1 and 0.2 where $\tau=\hat{\beta}^{2} t$. The initial radius is $a(0)=1$. Note that with such small interface displacements the Rossby number is small and so the use of the Cushman-Roisin et al. (1990) formula is valid. Note that initially $a b=0.69$ for the $\lambda=0.1$ cyclone and $a b=1.0$ for the $\lambda=0.2$ cyclone. This puts them in a parameter regime such that the approximation that most of the radiated energy occurs in the gravest mode, detailed in the previous section, is valid (see also table 1) and hence consistent with the initial assumption that $|v / u| \ll 1$. Moreover, since $a$ decreases with time, this approximation gets better with time. The weaker $(\lambda=0.1)$ cyclone in figure $4(b)$ decays more rapidly than the stronger cyclone $(\lambda=0.2)$ in figure $4(a)$. The decay in radius appears to be exponential-like. In fact, in the 

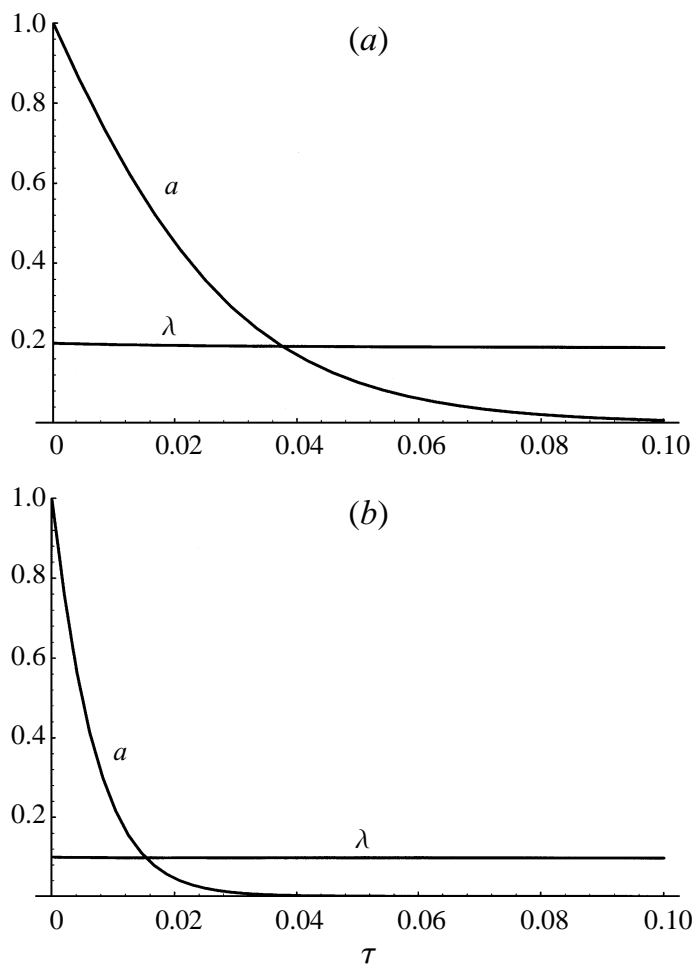

FIGURE 4. ( $a$ ) Evolution of the cyclone radius $a$ and amplitude $\lambda$ as a function of $\tau$ in response to the radiative energy loss. Here $a(0)=1$ and $\lambda(0)=0.2$. (b) As in $(a)$, but for weaker initial amplitude $\lambda(0)=0.1$. Note that in this case the decay time for the radius $a$ is shorter than the stronger cyclone in $(a)$.

limit $a \ll 1$ it is precisely exponential decay. To see this, observe that for small $a, Y_{2}(a b) \sim-4 /\left(\pi a^{2} b^{2}\right)$ and $Y_{1}(a b) \sim-2 /(\pi a b)$. Thus, to leading order in $a$, (42) becomes

$$
\frac{\mathrm{d} a}{\mathrm{~d} \tau}=-\frac{8 a b}{\lambda^{2}\left(1+b^{2}\right)^{3}},
$$

which yields an exponential decay law for $a$ with e-folding time equal to $\lambda^{2}(1+$ $\left.b^{2}\right)^{3} / 8 b=\lambda^{3 / 2}(2-\lambda)^{-5 / 2}$. This is a monotone decreasing function of $\lambda$ on the interval $[1,0]$, reflecting the fact that weaker cyclones (smaller $\lambda$ ) decay more rapidly than stronger cyclones.

Dimensionally, choosing a typical value relevant to oceanic vortices of $\hat{\beta}=0.006$ and recalling that the dimensional timescale is $f^{-1}$ (choosing $f=8 \times 10^{-5} \mathrm{~s}^{-1}$ ) implies that $\tau=1$ is equivalent to about 4000 days. Figure $4(a, b)$ indicates that cyclones undergo significant decay (i.e. decaying to half their original size) in 80 days $(\tau=0.02)$ for the stronger cyclone and in 25 days $(\tau=0.006)$ for the weaker cyclone. Thus the radiative decay mechanism explored here may be equally, or indeed more, significant to other non-conservative decay processes such as friction or mixing.

Typical parameters relevant to the Jovian system are (see e.g. Williams \& Yamagata 1984) $\beta=4 \times 10^{-9} \mathrm{~km}^{-1} \mathrm{~s}^{-1}, L=1500 \mathrm{~km}$ and $f=2 \times 10^{-4} \mathrm{~s}^{-1}$ giving $\hat{\beta}=0.03$. For this case $\tau=1$ corresponds to about 60 (Earth) days, implying that the decay 
of cyclones is much more rapid on Jupiter. Although the presence of significant anticyclonic zonal shear flows on Jupiter make it debatable whether the present theory is directly applicable to such vortices.

\section{Conclusion}

Analytic solutions have been found for the streamfunction field exterior to the separatrix for finite-amplitude shallow water cyclones and anticyclones. This calculation is quite general and depends only on the zonal drift velocity of the vortex and the fact that a circular separatrix exists in the geostrophic streamfunction field in the frame of reference moving with the vortex. It does not require that the vortex amplitude be vanishingly small compared to the mean layer thickness (i.e. quasi-geostrophic theory) but is valid for finite vortex amplitudes. Indeed this is a necessary requirement, so that the vortices drift westward at an amplitude-dependent speed, different from the long Rossby wave speed. In the case of anticyclones, which drift westward faster than all possible Rossby wave phase speeds, the exterior field is evanescent and steady solutions are possible. This is not so for cyclones, because they drift westward at a speed which matches an available Rossby wave phase speed. A quasi-steady assumption along with a radiation condition enables the exterior streamfunction field to be calculated analytically. The energy flux carried by these Rossby waves is then found and, further, by assuming that most of the radiated energy occurs in the lowest-order mode an expression for the northward drift velocity of the cyclone is obtained. This expression reduces to that obtained by Korotaev \& Fedotov (1994) in the barotropic limit.

A general method of determining the response of cyclones to Rossby wave radiation is presented based on relating the loss of energy in the Rossby wave wake to the rate of change of energy of the cyclone and using the conservation of potential vorticity for the central fluid column of the vortex. An example calculation for a top-hat cyclonic vortex shows that the primary response to the radiation is a decrease in vortex radius. This implies that mass must be lost from the interior of the separatrix, and it is envisaged that this occurs in the vicinity of the stagnation point as in Korotaev \& Fedotov (1994). In particular, they showed numerically that the resulting vortex sheet affects some aspects of the vortex evolution, primarily its trajectory. It is probable that the situation in the shallow water case here is similar and this would require numerical investigation. The shallow water numerical experiments on $\beta$-plane cyclones by Davey \& Killworth (1984) also show evidence for mass leakage and subsequent decrease in radius. Moreover, consistent with the findings here, they show that stronger cyclones are more robust. It is arguable, however, whether it is fair to make a direct comparison with the results of Davey \& Killworth (1984) since it is possible that the behaviour they observe is due to the initial adjustment of an initially stationary eddy. Also, their experiments were run only the relatively short time of about 200 inertial periods, or about 20 days, which is insufficient to observe significant decay in strong cyclones by the mechanism proposed here.

The theoretical estimate for the timescale for the cyclone to decay to half its initial radius obtained here (e.g. 60-120 days, depending on the initial amplitude of the cyclone) is of possible relevance to oceanic vortices. In particular, the Rossby wave radiation mechanism explored here may serve to explain the observed oceanic bias towards greater numbers of anticyclones rather than cyclones. 
Benilov, E. S. 1996 Beta-induced translation of strong isolated eddies. J. Phys. Oceanogr. 26, $2223-2229$.

Cushman-Roisin, B. 1986 Frontal geostrophic dynamics. J. Phys. Oceanogr. 16, 132-143.

Cushman-Roisin, B., Chassignet, E. P. \& Tang, B. 1990 Westward motion of mesoscale eddies. $J$. Phys. Oceanogr. 20, 758-768.

Cushman-Roisin, B. \& TANG, B. 1990 Geostrophic turbulence and emergence of eddies beyond the radius of deformation. J. Phys. Oceanogr. 20, 97-113.

Davey, M. K. \& Killworth, P. D. 1984 Isolated waves and eddies in a shallow water model. $J$. Phys. Oceanogr. 14, 1047-1064.

FLIERL, G. R. 1984 Rossby wave radiation from a strongly nonlinear warm eddy. J. Phys. Oceanogr. 14, 47-58.

Flierl, G. R. \& Haines, K. 1994 The decay of modons due to Rossby wave radiation. Phys. Fluids 6, 3487-3497.

Korotaev, G. K. \& Fedotov, A. B. 1994 Dynamics of an isolated barotropic eddy on a $\beta$-plane. $J$. Fluid Mech. 264, 277-301.

McWilliams, J. C. 1985 Submesoscale, coherent vortices in the ocean. Rev. Geophys. 23, 165-182.

Miles, J. W. 1968 Lee waves in a stratified flow. Part 2. Semicircular obstacle. J. Fluid Mech. 33, 803-814.

Nezlin, M. V. \& Sutyrin, G. G. 1994 Problems of simulation of large long-lived vortices in the atmospheres of the giant planets (Jupiter, Saturn, Neptune). Surveys Geophys. 15, 63-99.

NyCANDER, J. 1994 Steady vortices in plasmas and geophysical flows. Chaos 4, 253-267.

Polvani, L. M., McWilliams, J. C., Spall, M. A. \& Ford, R. 1994 The coherent structures of shallow water turbulence: Deformation radius effects, cyclone/anticyclone asymmetry and gravity-wave generation. Chaos 4, 177-186.

ReZniK, G. M. 1992 Dynamics of singular vortices on a beta-plane. J. Fluid Mech. 240, 405-432.

Sutyrin, G. G., Hesthaven, J. S, Lynov, J. P. \& Rasmussen, J. J. 1994 Dynamical properties of vortical structures on the $\beta$-plane. J. Fluid Mech. 268, 103-131.

Swaters, G. E. 1994 On stationary equivalent modons in an eastward flow. Phys. Fluids 6, 118-123.

Swaters, G. E. \& Flierl, G. R. 1991 Ventilated coherent cold eddies on a sloping bottom. J. Fluid Mech. 223, 565-587.

Williams, G. P. \& Yamagata, T. 1984 Geostrophic regimes, intermediate solitary vortices and Jovian eddies. J. Atmos. Sci. 41, 453-478. 